\title{
The direction-selective contrast response of area 18 neurons is different for first- and second-order motion
}

\author{
TIMOTHY LEDGEWAY, CHANG'AN ZHAN, AARON P. JOHNSON, YUNING SONG, \\ AND CURTIS L. BAKER, JR. \\ McGill Vision Research, Department of Ophthalmology, McGill University, Montreal, Quebec, Canada
}

(Received September 5, 2004; AcCEPTED December 8, 2004)

\begin{abstract}
Cortical neurons selective for the direction of motion often exhibit some limited response to motion in their nonpreferred directions. Here we examine the dependence of neuronal direction selectivity on stimulus contrast, both for first-order (luminance-modulated, sine-wave grating) and second-order (contrast-modulated envelope) stimuli. We measured responses from single neurons in area 18 of cat visual cortex to both kinds of moving stimuli over a wide range of contrasts (1.25-80\%). Direction-selective contrast response functions (CRFs) were calculated as the preferred-minus-null difference in average firing frequency as a function of contrast. We also applied receiver operating characteristic analysis to our CRF data to obtain neurometric functions characterizing the potential ability of each neuron to discriminate motion direction at each contrast level tested. CRFs for sine-wave gratings were usually monotonic; however, a substantial minority of neurons $(35 \%)$ exhibited nonmonotonic CRFs (such that the degree of direction selectivity decreased with increasing contrast). The underlying preferred and nonpreferred direction CRFs were diverse, often having different shapes in a given neuron. Neurometric functions for direction discrimination showed a similar degree of heterogeneity, including instances of nonmonotonicity. For contrast-modulated stimuli, however, CRFs for either carrier or envelope contrast were always monotonic. In a given neuron, neurometric thresholds were typically much higher for second- than for first-order stimuli. These results demonstrate that the degree of a cell's direction selectivity depends on the contrast at which it is measured, and therefore is not a characteristic parameter of a neuron. In general, contrast response functions for first-order stimuli were very heterogeneous in shape and sensitivity, while those for second-order stimuli showed less sensitivity and were quite stereotyped in shape.
\end{abstract}

Keywords: Visual cortex, Direction selectivity, Contrast response function, First-order motion, Second-order motion

\section{Introduction}

Neurons in the early visual cortex areas of cats and monkeys are often selective to stimulus orientation and direction of motion (Hubel \& Wiesel, 1968), and also spatial and temporal frequency of sinusoidal luminance gratings (e.g., Holub \& Morton-Gibson, 1981; Foster et al., 1985). Striate cortical neuron responses typically show a monotonic rather than tuned response to increasing contrast, often with a rapid initial rise followed by compression and saturation (e.g., Cooper \& Robson, 1968; Movshon \& Tolhurst, 1975; Dean, 1981; Albrecht \& Hamilton, 1982). However, at high contrast levels some area 17 neurons show a pronounced decline in response ("supersaturation"), producing a nonmonotonic function of contrast (e.g., Albrecht \& Hamilton, 1982; Li \& Creutzfeldt, 1984; Bonds, 1991; Somers et al., 2002) reminiscent

Address correspondence and reprint requests to: Timothy Ledgeway, School of Psychology, University of Nottingham, University Park, Nottingham, NG7 2RD UK. E-mail: timothy.ledgeway@nottingham.ac.uk of the sound intensity "tuning" in primary auditory cortex neurons (Brugge \& Merzenich, 1973; Phillips et al., 1994).

A neuron's contrast response function (CRF) is important because nonlinearities in its shape affect stimulus selectivity and other aspects of efficient sensory coding. For example, an expansive (convex) function substantially sharpens tuning (Albrecht \& Geisler, 1991; Heeger, 1992a). The potential degradation of selectivity by saturation (Robson, 1975) is largely prevented by a contrast gain control in striate cortex, which preserves the CRF shape for nonoptimal stimuli (Albrecht \& Hamilton, 1982; Skottun et al., 1987; Albrecht \& Geisler, 1991; Bonds, 1991; Geisler \& Albrecht, 1992; Carandini \& Heeger, 1994). The CRF shape also has theoretical implications for efficiency of sensory coding (Simoncelli, 2003), both for enhancement of sparse coding (Olshausen \& Field, 2004) and for ensuring efficient distribution of firing rates in response to natural images (Laughlin, 1981; Baddeley et al., 1998).

Direction selectivity is of particular interest in this regard, since differences in CRFs for preferred versus nonpreferred directions could result in the net directionality being contrast dependent and 
even nonmonotonic, as demonstrated by $\mathrm{Li}$ and Creutzfeldt (1984) using moving bar stimuli. [Indeed some human psychophysical studies of motion perception have demonstrated nonmonotonic dependence on contrast. (Derrington \& Goddard, 1989; Cleary, 1990)]. If direction selectivity is contrast dependent, then its measurement made at a single contrast should not be considered a characteristic parameter of a cortical neuron.

Stimuli defined by differences in derived properties such as contrast or texture ("second-order" stimuli—Chubb \& Sperling, 1988; Cavanagh \& Mather, 1989) also drive similarly stimulusselective responses in many early visual cortical neurons in cat areas 17/18 (Zhou \& Baker, 1993; Leventhal et al., 1998) and primate V1 (Chaudhuri \& Albright, 1997). While neurons often respond more weakly to these second-order motion stimuli (e.g., Zhou \& Baker, 1994) than to conventional luminance-defined ("first-order") bars or gratings, previous measurements of CRFs for these complex stimuli (O'Keefe \& Movshon, 1998) examined neurons in area MT/V5 rather than early visual areas, and did not measure direction selectivity. Using a second-order stimulus composed of a stationary high spatial-frequency grating (carrier) that was contrast modulated by a drifting sine-wave grating, area 18 neurons often showed optimal spatial and temporal frequencies distinct from those for luminance gratings, and preferences for much higher carrier frequencies (Mareschal \& Baker, 1999). These findings suggested that such cells receive converging inputs from two independent processing streams, specialized for encoding either first- or second-order motion (for review see Baker, 1999). Thus, a neuron could exhibit qualitatively different CRFs to the two kinds of motion stimuli, which might shed light on underlying mechanisms as well as functions of the two processing streams.

Here we address these issues by measuring neurons' responses to both first- and second-order motion stimuli over a wide range of stimulus contrasts, in cat area 18 where about half of the cells are responsive to both kinds of stimuli. We also apply receiver operating characteristic (ROC) analysis (e.g., Tolhurst et al., 1983; Parker \& Newsome, 1998) to quantitatively characterize the potential motion-direction information provided by individual neurons.

\section{Materials and methods}

\section{Animal preparation}

Procedures for anesthesia, surgery, life support, and maintenance of optical integrity were essentially the same as described previously (Mareschal \& Baker, 1998). Surgical anesthesia was induced using halothane/oxygen and maintained with i.v. thiopentone sodium. Following surgery the animal was paralyzed with gallamine triethiodide; maintenance anesthesia and paralysis were provided by oxygen/nitrous oxide and i.v. infusion of sodium pentobarbital and gallamine. Core body temperature, expired $\mathrm{CO}_{2}$, blood $\mathrm{O}_{2}$, heart rate, and electroencephalogram (EEG) were continuously monitored and maintained at appropriate levels. Extracellular spike recordings from area 18 were obtained with platinum-iridium microelectrodes (Frederick Haer, Brunswick, ME); the dura was protected with $2 \%$ agarose (Sigma Chemical Co., St. Louis, MO) covered by petroleum jelly. Corneal protection during surgery was provided with topical carboxymethylcellulose drops (1\%) and subsequently with contact lenses; spectacle lenses and artificial pupils produced emmetropia at a viewing distance of $57 \mathrm{~cm}$. All animal procedures were approved by the Animal Care Committee of McGill University and are in accordance with the guidelines of the Canadian Council on Animal Care.
Visual stimuli and spike collection

For quantitative experiments, all visual stimuli were generated on a Macintosh (G4 1000 MHz) using Psychophysics Toolbox (Brainard, 1997; Pelli, 1997) and custom software written in Matlab (Mathworks) and C. Stimuli were displayed on a CRT screen (NEC FP1350, $640 \times 480,75 \mathrm{~Hz}, 36 \mathrm{~cd} / \mathrm{m}^{2}$ ) which was $z$-linearized by gamma-corrected lookup tables.

Visual stimuli consisted of drifting periodic patterns enclosed in circular, or occasionally square, windows. The first stimulus patterns employed were conventional (luminance-modulated) sinewave gratings:

$$
L(x, y, t)=L_{0}\left\{1+C \sin \left[2 \pi f_{x}(x \cos \theta+y \sin \theta)-2 \pi f_{t} t\right]\right\},
$$

where $L_{0}$ is mean luminance, $C$ is Michelson contrast, $f_{x}$ is spatial frequency, $f_{t}$ is temporal frequency, and $\theta$ is orientation.

Contrast envelope stimuli (Mareschal \& Baker, 1999) consisted of a static carrier pattern whose amplitude was modulated by a drifting envelope pattern:

$$
\begin{aligned}
L(x, y, t)=L_{0} & \left\{1+C_{c} \sin \left[2 \pi f_{c}\left(x \cos \theta_{c}+y \sin \theta_{c}\right)\right]\right. \\
& \left.*\left(1+C_{e} \sin 2 \pi\left(f_{e}\left(x \cos \theta_{e}+y \sin \theta_{e}\right)-f_{t} t\right)\right) / 2\right\},
\end{aligned}
$$

where the carrier was a high spatial frequency $\left(f_{c}\right)$ grating with orientation $\theta_{c}$ and contrast $C_{c}$, and the envelope was a low spatial frequency $\left(f_{e}\right)$ grating with orientation $\theta_{e}$ and contrast $C_{e}$, drifting at a temporal frequency of $f_{t}$. Examples of contrast envelope stimuli for varying values of $C_{c}$ and $C_{e}$ will be shown later. A uniform gray level $\left(L_{0}\right)$ was presented outside the display window, and also across the entire screen at all times between stimulus presentations.

Times of spikes were collected (100- $\mu$ s accuracy) via a lab interface (Instrutech, ITC-18, New York, NY) on the same host computer. Preliminary data analyses were plotted on a second console screen for immediate assessment to guide subsequent measurements.

\section{Electrophysiology}

Amplified microelectrode signals were fed to an audio monitor, oscilloscope, and window discriminator (Frederick Haer). Adequacy of isolation was also monitored by display of detected spike waveforms on a digital oscilloscope with delayed triggering. A manually controlled slit projector was used to ascertain the dominant eye, approximate receptive-field position, eccentricity, and optimal orientation; the nondominant eye was subsequently occluded.

Computer-controlled stimuli were used for quantitative measurements. Drifting gratings (contrast $=30 \%$ ) were used to accurately measure optimal orientation, spatial frequency, temporal frequency, and direction of motion. The neuron's receptive-field position center was then mapped quantitatively using briefly presented, small patches of optimal grating. With the optimal grating centered on the optimal position, a series of circular window sizes were tested to determine the minimal size to optimally drive the neuron. With all these stimulus parameters thus optimized, the neuron's direction-selective contrast response function was measured by presenting gratings at a series of contrasts $(1.25-80 \%$ in octave steps), for both preferred and nonpreferred directions of motion, in a random order, with the full set of conditions repeated 
20 times; also included were blank screen controls (contrast $=0$ ). Spike responses to each presentation were recorded for $3 \mathrm{~s}$, with an intertrial interval of $c a$. 1-3 s.

Some neurons were also assessed for response to contrast envelope stimuli [eqn. (2)], using a protocol like that described previously (Mareschal \& Baker, 1999). Starting with envelope parameters like those of the optimal grating, and high contrast values $\left(C_{e}=100 \%, C_{c}=70 \%\right)$, a range of carrier spatial frequencies were tested; a neuron was used for subsequent envelope response measurements only if it exhibited a clear bandpass tuning to carrier spatial frequencies much higher than, and well outside, the measured spatial-frequency response to simple gratings (Zhou \& Baker, 1994; Mareschal \& Baker, 1998). Using the measured optimal carrier spatial frequency, other envelope stimulus parameters were adjusted to maximize response. Following the same procedure as for simple gratings, the neuron's response to carrier contrast $\left(C_{c}\right)$ of envelope stimuli was measured, at a fixed value of envelope contrast $\left(C_{e}=100 \%\right)$. On some neurons the response to varying envelope contrast was also measured, keeping carrier contrast fixed at $70 \%$.

\section{Data analysis}

Responses were plotted as cumulative poststimulus time histograms (bin width $10 \mathrm{~ms}$ ) for each stimulus condition tested. Neurons were classified as simple or complex type based on the ratio of modulated to unmodulated responses to the neuron's optimal grating (Skottun et al., 1991). These data were summarized in plots of time-averaged spike frequency as a function of the stimulus parameter being varied, to determine optimal stimulus parameter values. Average responses to the blank screen condition provided estimates of the spontaneous activity, which was typically negligible and for clarity is not shown in the example plots in the figures.

Contrast response was quantified as average spike frequency, measured as a function of stimulus contrast ( $C$ for sine-wave gratings, $C_{c}$ or $C_{e}$ for contrast envelopes), separately for stimuli drifting in the neuron's preferred and nonpreferred directions of motion. The direction-selective contrast response was quantified as the difference of the preferred and non-preferred direction responses at each contrast. This measure was chosen in preference to ratio-based measures to avoid problematic behaviors at very low contrasts (excessive variance and responses tending to zero). In addition, neurometric functions for direction-selective contrast responses were also plotted from these data, as will be described later.

Key aspects of these CRFs were parameterized by a few scalar indices. An indicator of the overall responsivity of the neuron was taken as the spike frequency to the neuron's optimal grating, moving in the preferred direction.

An important result, demonstrated below, was that some neurons exhibited a nonmonotonic dependence of direction selectivity on contrast. The degree to which this behavior was exhibited was quantified by a dimensionless monotonicity index $(M I)$ :

$$
M I=1.0-\left(R_{\max }-R_{\text {contrast } \max }\right) /\left(R_{\max }-R_{\min }\right),
$$

where $R_{\text {contrast max }}$ is the response strength at the maximal contrast tested (typically $80 \%$ ), and $R_{\max }$ and $R_{\min }$ are the maximal and minimal values of the contrast response. For a monotonic CRF, the $M I$ will be unity, but nonmonotonic behavior will result in values less than one.
As an additional shape descriptor of contrast response functions, the degree to which response saturated at high contrasts was estimated as the contrast saturation index $(S I)$ taken as the slope of the function at the high contrast end:

$$
S I=\left(R_{\text {contrast max }}-R_{\text {contrast half-max }}\right) / R_{\max },
$$

where $R_{\text {contrast max }}$ and $R_{\text {contrast half-max }}$ are the response strengths at the maximal (typically 80\%) and half the maximal contrast tested, respectively. This index, also dimensionless, will be positive for a monotonically increasing, nonsaturating response, zero for an asymptotic saturation, and negative for a non-monotonic response.

\section{Results}

Quantitative measurements were obtained from 58 neurons recorded in 22 cats, in most cases in parallel with other concurrent studies (Zhan et al., 2002; Zhan \& Baker, 2003; Song \& Baker, 2003) conducted in the same animals.

\section{Contrast response to first-order motion (drifting gratings)}

Contrast response was quantified as time-averaged spike frequency, plotted as a function of stimulus contrast. In Fig. 1, the CRFs of six neurons to motion in both the preferred $(\mathrm{P}$, filled circles) and nonpreferred ( $\mathrm{N}$, unfilled circles) directions (left column) and also the $\mathrm{P}-\mathrm{N}$ difference (right column) are plotted to illustrate the variety of neuronal response patterns. When tested with motion in the P-direction, the response amplitude for most cortical cells initially increases, and then for some neurons (B \& E) exhibits rapid compression or even saturation at higher contrasts. However, some neurons (A \& C) display little or no saturation in the P-direction whilst others (D \& F) exhibit clear supersaturation (peak response at an intermediate contrast followed by a decline at higher levels). For many neurons, the $\mathrm{N}$-direction response pattern has a qualitatively different shape to that for the P-direction. Comparing the difference in $\mathrm{P}$ and $\mathrm{N}$ responses at each contrast (rightmost column) reveals that the degree of direction selectivity is highly contrast dependent for many neurons. For the cells shown in Figs. $1 \mathrm{~A}-1 \mathrm{C}$, the $\mathrm{P}-\mathrm{N}$ difference is monotonic with contrast, showing either a quantitative increase in direction selectivity as contrast increases over the whole range, or else some degree of saturation at the highest contrasts. For the remaining three neurons (Figs. 1D-1F) the P-N $\mathrm{CRF}$ is nonmonotonic, such that the difference in firing rate between the two opposing directions is initially small, then increases over some range before declining sharply at the highest contrasts.

Two indices of function shape were used to characterize all the $\mathrm{P}-\mathrm{N}$ CRFs for which complete quantitative data were available $(n=49)$. The monotonicity index [MI, eqn. (3)] for this sample had a mean of $0.89(\mathrm{SD}=0.21)$, with a sizeable proportion of cells (35\%) showing nonmonotonic $\mathrm{P}-\mathrm{N}$ contrast response functions. The saturation indices [SI, eqn. (4)] were distributed approximately normally around a mean of $0.12(\mathrm{SD}=0.48)$, indicating that most neurons exhibited some degree of $\mathrm{P}-\mathrm{N}$ response saturation.

ROC analysis and neurometric functions to first-order motion stimuli

While the above analyses dealt with average $\mathrm{P}, \mathrm{N}$, and $\mathrm{P}-\mathrm{N}$ responses, they do not address the extent to which a neuron 
(A)

(B)

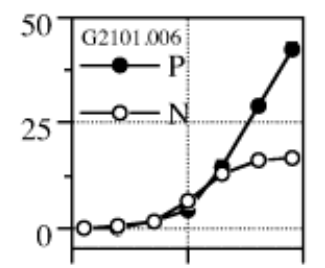

(D)

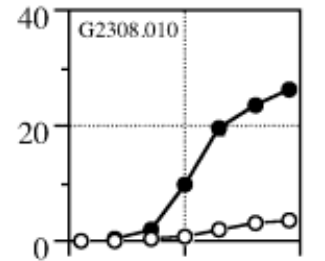

(C)

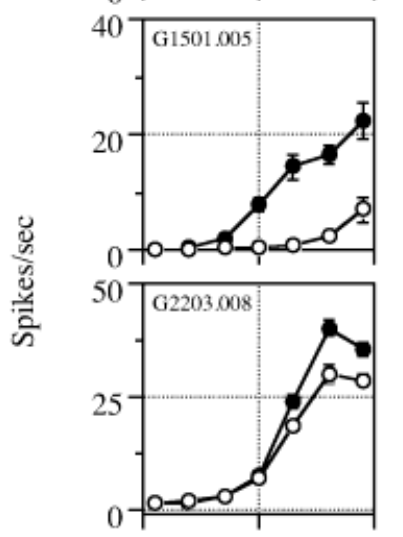

(E)

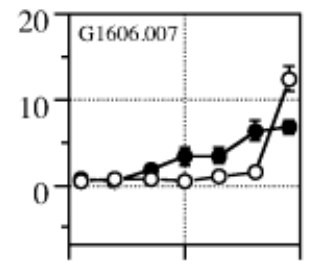

(F)

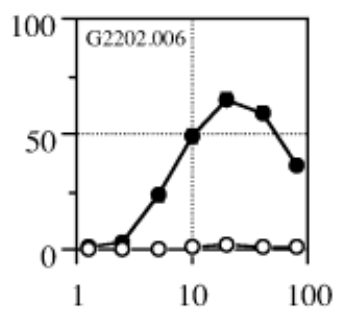

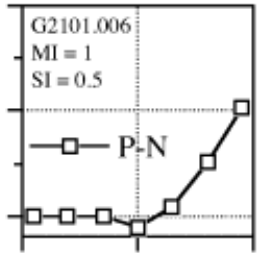
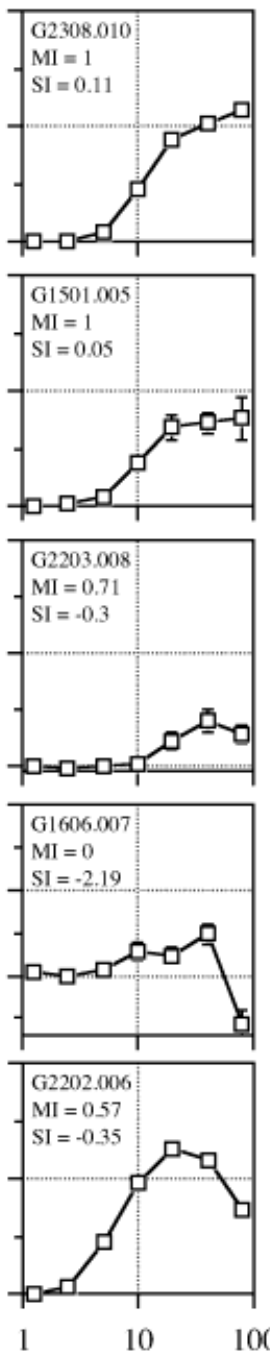

Grating contrast $(C)$
Fig. 1. Contrast response functions (CRFs) of time-averaged spike frequency to sine-wave gratings for six representative neurons. Left-hand panels show responses in each neuron's preferred ( $\mathrm{P}$, filled circles) and nonpreferred $(\mathrm{N}$, unfilled circles) directions of motion; panels on the right show the $\mathrm{P}-\mathrm{N}$ difference (unfilled squares), indicating direction selectivity as a function of contrast. (A-C) Three neurons with monotonic CRFs. (D-F) Three neurons whose directionselective CRFs are nonmonotonic, for differing reasons in the underlying $\mathrm{P}$ and $\mathrm{N}$ responses. provides statistically reliable information for motion-direction discrimination at different contrast levels. Since the variance of neural firing rate can interact with its mean in a complex manner (e.g., Tolhurst et al., 1983; Sachs \& Baker, 2003), a neuron's discriminatory power may not be straightforwardly related to its average response. To address this issue, we employed nonparametric procedures of neurometric function analysis, which are conventional (Barlow et al., 1971; Tolhurst et al., 1983; Britten et al., 1992; Parker \& Newsome, 1998) and will be described here only briefly.

For each contrast condition and motion direction, a histogram was formed of the neuron's responses (spike counts over the 3-s stimulus duration) across the individual trials. Examples of such histograms for three contrast levels are shown in Fig. 2A, which progressively separate with increasing contrast - that is, for a very low contrast $(2.5 \%)$ the histograms for $\mathrm{P}$ (filled bars) and $\mathrm{N}$ (unfilled bars) directions overlap completely, at an intermediate level $(5 \%)$ they overlap only partially, and at a higher contrast $(10 \%)$ they are entirely distinct.

To characterize the discriminability of these pairs of distributions at each contrast, a plot was constructed of the estimated probability that the P-direction response exceeded some criterion value, against the probability that the response in the $\mathrm{N}$-direction surpassed the same criterion. Each such "receiver operating characteristic" (ROC) curve was parametric in the criterion value, which ranged from zero to the maximum possible firing rate. Fig. 2B shows three such ROC curves corresponding to the pairs of response distributions in Fig. 2A. The area beneath each ROC curve corresponds to the expected percentage of trials that a statistically "ideal observer" would perform correctly (Barlow et al., 1971). These theoretical percentage correct values plotted 
(A)
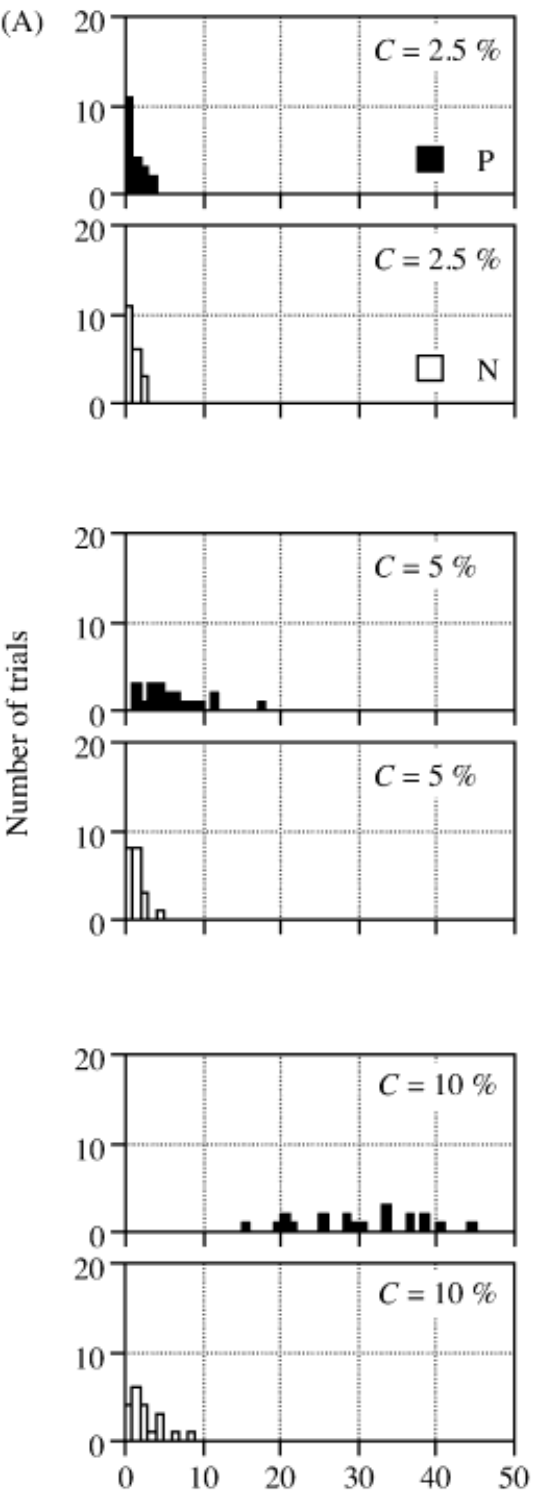

Spike count
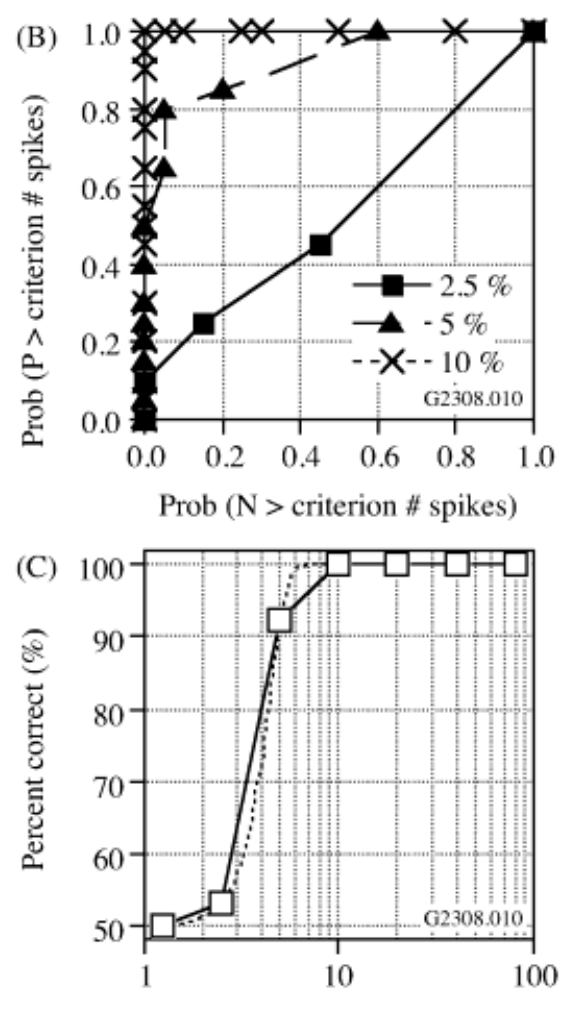

Grating contrast $(C)$

Fig. 2. Example of neurometric function measurement, for the same neuron as in Fig. 1B. (A) Histograms of number of trials (out of a total of 20) in which the neuron's response (total numbers of spikes) fell within varying ranges, for three contrast levels; filled and unfilled vertical bars indicate results for the preferred and nonpreferred directions, respectively. Response distributions are identical at a low contrast (top), overlapping at an intermediate contrast (middle), and completely separated at a higher contrast (bottom). (B) Receiver operating characteristic (ROC) curves for the three contrasts in A, plotting the estimated probability that the preferred-direction response exceeds a given criterion, against that for the nonpreferred response. Each point represents a different criterion. (C) Neurometric function (unfilled squares) of percent correct direction identification, calculated from the areas under the ROC curves, as a function of contrast for all seven contrast levels tested. The dotted line shows the best-fitting Weibull function [eqn. (5), $\alpha=4.09, \beta=4.92]$.

against contrast provides a "neurometric function" (Fig. 2C), in some ways analogous to a behavioral psychometric function, characterizing the neuron's direction-identification performance.

The neurometric data were further summarized by fitting a modified Weibull (1951) function (dotted line in Fig. 2C) of the form:

$$
P_{c}=100-50 * 2^{z}
$$

where $P_{c}$ is percent correct, $z=-(C / \alpha)^{\beta}, C$ is stimulus contrast (percent), $\alpha$ determines position along the contrast axis, and $\beta$ specifies the slope. The value $\alpha$, corresponding to $75 \%$ correct, was taken as a scalar measure of neural contrast threshold. Weibull functions were fit only to neurometric data where the percent correct performance for an individual neuron clearly exceeded $75 \%$ correct. Whenever this conservative criterion was not satisfied (indicating that the cell did not exhibit a sufficiently reliable
$\mathrm{P} v s . \mathrm{N}$ differential response at any contrast), the cell was excluded from further analyses. When the neurometric function was itself nonmonotonic, the Weibull fit was constrained to utilize only the initial monotonic portion of the data.

Fig. 3 shows neurometric functions for the same six neurons as in Fig. 1 together with the corresponding Weibull fits (dotted lines) and $M I$ values computed from the neurometric data. Although for the majority of cells (e.g., A, B, \& E) the shape of the P-N CRF was similar to the neurometric function (i.e., either both were monotonic or both nonmonotonic), for a number of neurons (e.g., $\mathrm{C}, \mathrm{D}, \& \mathrm{~F})$ this was not the case. For example, the cell shown in Fig. $1 \mathrm{C}$ exhibited a monotonic $\mathrm{CRF}$ for the $\mathrm{P}-\mathrm{N}$ difference $(M I=$ 1.0 ) but its neurometric function (Fig. 3C) is clearly nonmonotonic $(M I=0.73)$, presumably reflecting the increased $\mathrm{N}$-direction response at the highest contrast and the proportionate increase of variance with mean response (Tolhurst et al., 1983). Conversely, for the cell shown in Fig. 1F the P-N CRF was highly nonmono- 
(A)

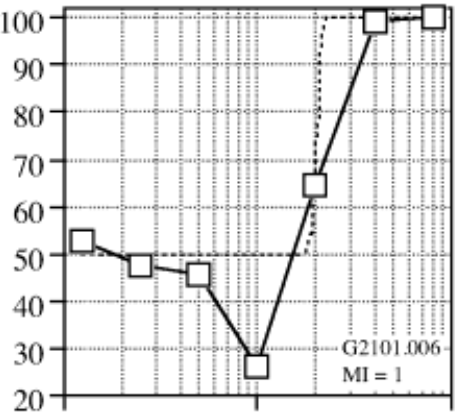

(B)

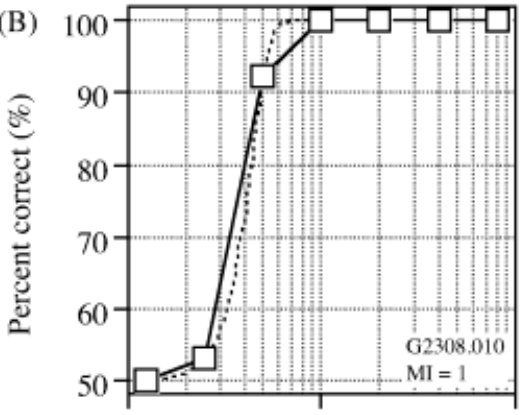

(C)

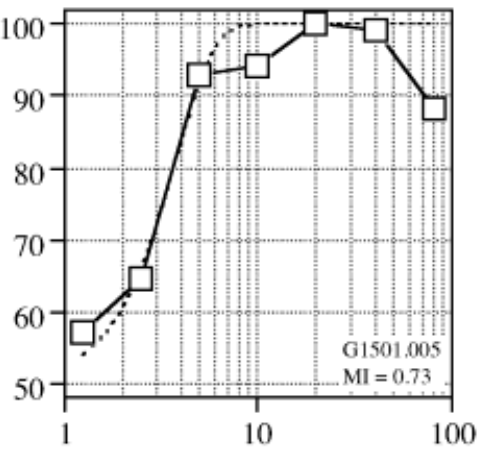

(D)

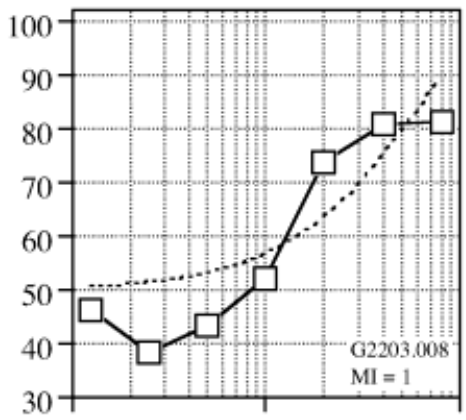

(E)

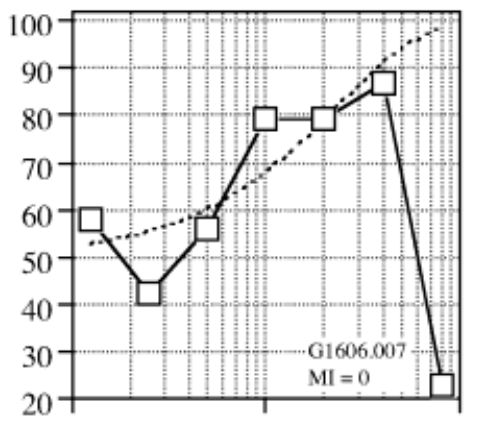

(F)

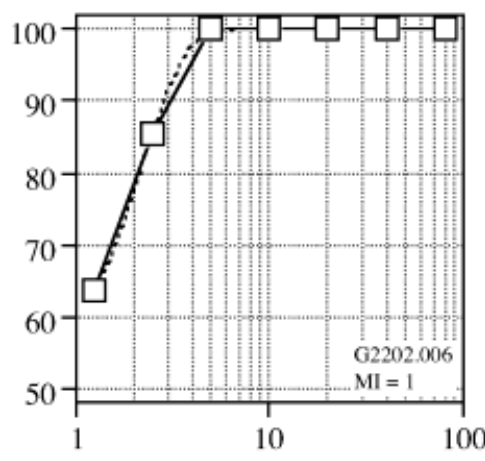

Fig. 3. Neurometric functions of direction identification for the same six neurons as in Fig. 1. In each case direction-identification performance (percent correct, unfilled squares) is plotted against contrast; dotted lines indicate the best-fitting Weibull functions. (A \& B) Neurons whose neurometric functions asymptote at perfect performance, but whose CRFs are nonsaturating (Figs. 1A \& 1B). Fitted Weibull parameters were, for A $(\alpha=20.5$, $\beta=27.89)$ and for $\mathrm{B}(\alpha=4.09, \beta=4.92)$. (C) Neuron exhibiting a nonmonotonic neurometric function, but a monotonic CRF (Fig. 1C); Weibull parameters were $\alpha=3.21, \beta=2.23$. (D) Neuron with modest directionality (Fig. 1D), and a correspondingly high neurometric threshold $(\alpha=39.5$, $\beta=1.13$ ). (E) Cell which was nonmonotonic in both kinds of response $(\alpha=15.64, \beta=0.97)$. (F) Neuron with monotonic, saturating neurometric performance, in spite of a nonmonotonic CRF (Fig. 1F) $(\alpha=1.85, \beta=1.97)$.

Grating contrast $(C)$

tonic $(M I=0.57)$, but since the cell gave almost no response to $\mathrm{N}$-direction motion the corresponding neurometric function shown in Fig. 3F was monotonic $(M I=1.0)$.

However, the average monotonicity index $(M I)$ of the neurometric functions was 0.9 ( $\mathrm{SD}=0.22)$, a value very similar to the mean $M I$ computed from the corresponding P-N CRFs. To quantify the relationship between these two indices, Fig. 4A shows a scatterplot of the $M I$ values computed from neurometric functions and from P-N CRFs for each neuron. For 28 (57\%) of the neurons, both $M I$ values were equal to unity (superimposed in the uppermost right corner of Fig. 4A) but for the remaining 21 cells (43\%) at least one of the $M I$ values was less than 1.0. The correlation between the two indices is positive and statistically significant [Spearman $\operatorname{rho}(49)=0.543 ; P<0.0001]$. The mean neural contrast threshold $(\alpha)$ was $19.58 \%(\mathrm{SD}=17.89 \%)$.
Supersaturation and the nonmonotonic dependence of direction selectivity on contrast

In principle, at least some of the response saturation and supersaturation effects could be a direct consequence of reaching a response ceiling in the preferred direction, due to the limited dynamic range of neural firing. To address this issue, we computed a saturation index $(S I)$ for each neuron analogous to that described by eqn. (4), but derived from the CRF for the preferred direction only. This provides a shape descriptor characterizing the extent to which the P-direction neuronal response tends to saturate or supersaturate with increasing contrast. As shown in Fig. 4B, the $S I$ values are clustered approximately uniformly around a value of zero, and there is no statistically significant correlation between the maximum response and the $S I$ for the preferred direction. Thus, 
(A)

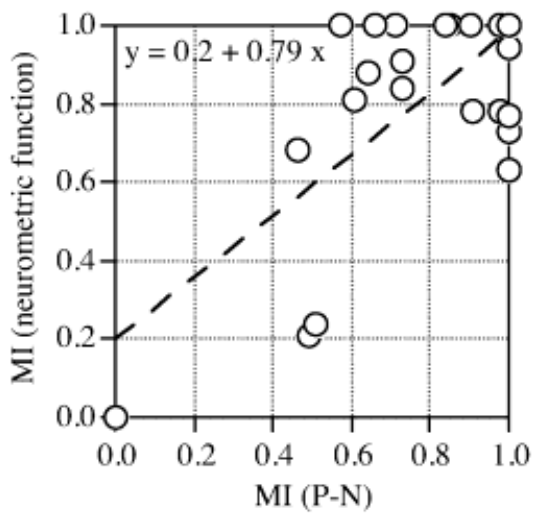

(B)

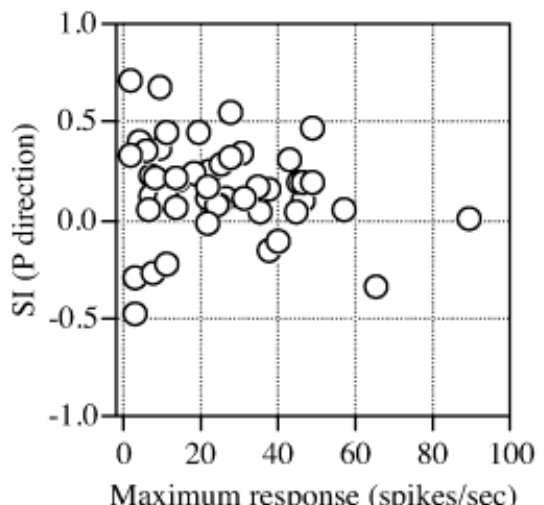

(C)

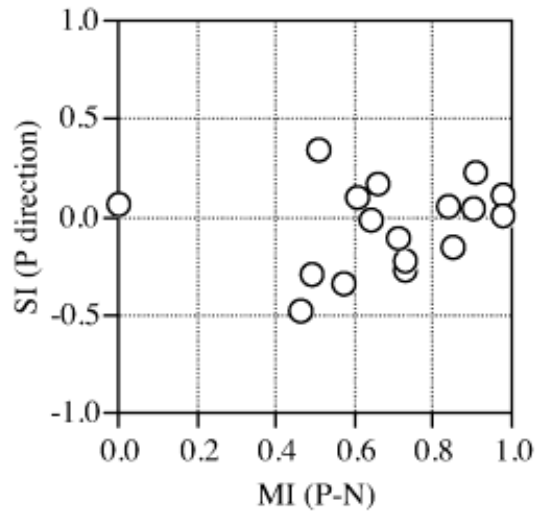

Fig. 4. Relationships between indices for CRF shape and maximum response. The three panels show scatterplots in which each point represents results from one neuron. (A) Relationship between monotonicity indices [eqn. (3)] from neurometric functions (ordinate) and direction-selective CRFs (abscissa). While many neurons showed differing degrees of monotonicity, the two measures were significantly correlated; the dashed line indicates linear regression. (B) Scatterplot of saturation indices [eqn. (4)] against maximum response (both computed for preferred direction only), showing that response saturation and supersaturation are uncorrelated with responsivity. (C) Scatterplot of saturation indices for preferred direction CRFs against monotonicity indices of direction-selective CRFs, for 17 neurons that exhibited a nonmonotonic dependence of directionality on contrast. Only ca. $50 \%$ of these cells show response supersaturation to optimal gratings drifting in the preferred direction of motion.

P-direction saturation or supersaturation $(S I \leq 0)$ is not simply found only in those neurons that are most strongly driven.

A related issue concerns the extent to which nonmonotonicity in the $\mathrm{P}-\mathrm{N}$ difference $\mathrm{CRF}$ is due to supersaturation in the
P-direction. Of the 17 cells exhibiting nonmonotonic P-N CRFs (i.e. $M I<1.0$ ), nine showed P-direction supersaturation but the remaining eight cells did not (Fig. 4C). For these latter neurons, a nonmonotonic $\mathrm{P}-\mathrm{N}$ CRF typically arises because the $\mathrm{N}$-direction response is recruited only at the highest contrasts where the P-direction response has already saturated, causing a decline in net $\mathrm{P}-\mathrm{N}$ difference. A striking example of this behavior is shown in Fig. 1E, where the N-direction response even exceeds the response in the P-direction at the highest contrast tested. Consequently, a nonmonotonic contrast dependence of direction selectivity is not always a simple result of P-direction supersaturation.

\section{Contrast response and neurometric function to} second-order motion (drifting contrast envelopes)

A subset of these neurons were also tested with second-order motion (contrast envelope stimuli), to which a total of 19 were responsive. Of these cells, 16 showed a sufficiently reliable differential response to $\mathrm{P}$ - versus $\mathrm{N}$-direction motion (i.e., a neural contrast threshold could be estimated from their neurometric functions) to warrant further quantitative analysis.

Fig. 5 shows CRFs for two representative neurons, including one shown previously in Fig. 1, to second-order motion in both the $\mathrm{P}$ (filled circles) and $\mathrm{N}$ (unfilled circles) directions (leftmost column) and also the $\mathrm{P}-\mathrm{N}$ difference (rightmost column). In Figs. 5A-5B, the contrast of the stationary carrier $\left(C_{c}\right)$ was varied whilst the envelope contrast was fixed at $100 \%$. For both cells the P-direction response was negligible over most of carrier contrast range tested, and then increased abruptly at the highest contrasts, with no evidence of either saturation or supersaturation. $\mathrm{N}$-direction responses were negligible even at the highest carrier contrasts. For all 16 cases examined, the $\mathrm{P}-\mathrm{N}$ CRFs were monotonic $(M I=1.0)$ and nonsaturating (mean $S I=0.83, \mathrm{SD}=0.22$ ).

Figs. 5C-5D show CRFs measured for the same two neurons when the envelope contrast $\left(C_{e}\right)$ was varied, with carrier contrast held constant $\left(C_{c}=70 \%\right)$. In all respects, the CRFs are comparable to those depicted in Figs. 5A-5B, with very little response at low to intermediate envelope contrasts, and significant P-direction responses only at the higher contrasts $\left(C_{e} \geq 50 \%\right)$. Again all the P-N CRFs $(n=7)$ were monotonic and nonsaturating (mean $S I=$ $0.71, \mathrm{SD}=0.33$ ). Indeed, the second-order motion CRFs for carrier and envelope contrast were remarkably similar in both shape and amplitude for all neurons examined.

ROC analysis was also employed to characterize each neuron's potential ability to discriminate second-order motion direction. The neurometric functions for both carrier and envelope contrast were always clearly monotonic and similar in shape to the corresponding P-N CRFs. The mean $M I$ of the neurometric functions was 0.995 ( $\mathrm{SD}=0.02$ ) when carrier contrast was varied and always 1.0 for varying envelope contrast. The mean neural threshold $(\alpha)$ for carrier contrast was $54.35 \%$ (SD $=16.94 \%$ ); the corresponding envelope contrast threshold was $66.39 \%$ (SD = $23.24 \%$ ).

These threshold values are relatively high compared to those obtained with first-order motion stimuli under equivalent conditions, and suggest that neuronal sensitivity is typically much lower to second-order than to first-order motion. Fig. 6A illustrates the relationship between carrier and grating contrast thresholds in 14 neurons for which both estimates were possible. The grating contrast thresholds exhibit greater variability (ranging from $c a$. $1-40 \%$ ) than those for the carrier, which are significantly lower [Wilcoxon $z_{(14)}=-3.296 ; P<0.001$ ] by about $1 \log$ unit on 
(A)

(B)

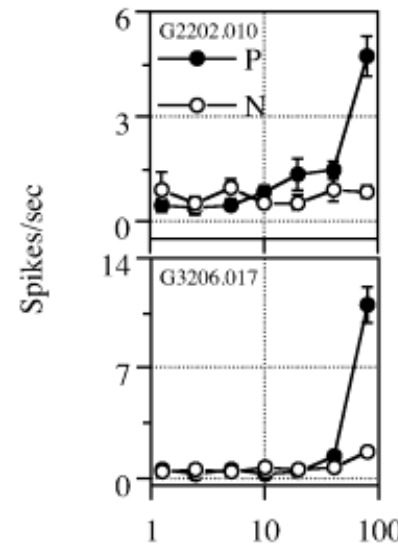

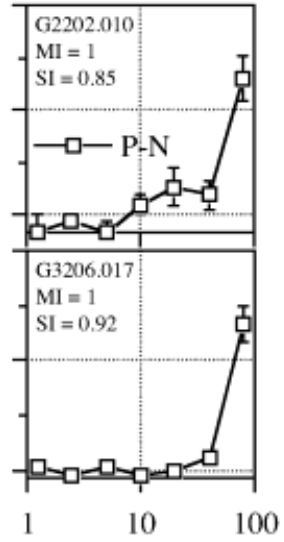

Carrier contrast $\left(C_{c}\right)$

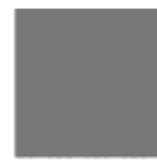

$1.25 \%$

(C)

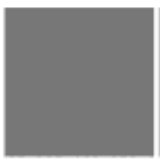

$2.5 \%$

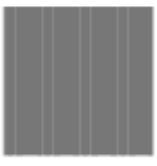

$5 \%$

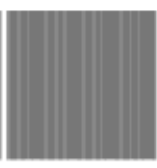

$10 \%$

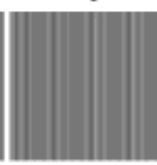

$20 \%$

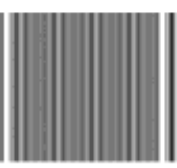

$40 \%$

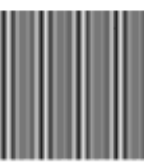

$80 \%$

D)
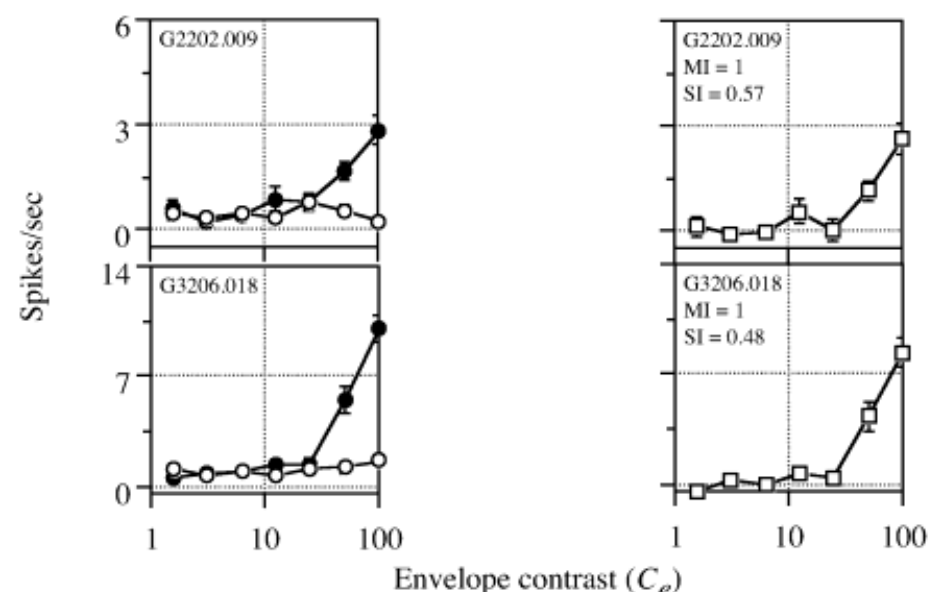

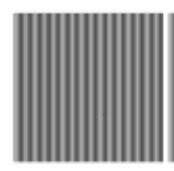

$1.56 \%$

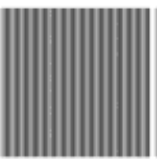

$3.13 \%$

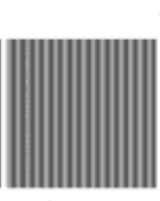

$6.25 \%$
Envelope contrast $\left(C_{e}\right)$

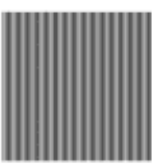

$12.5 \%$

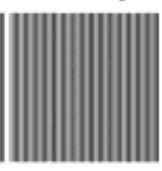

$25 \%$

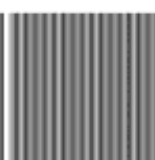

$50 \%$

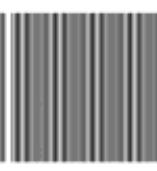

$100 \%$
Fig. 5. Contrast response functions (CRFs) of timeaveraged spike frequency to contrast envelope stimuli for two representative neurons. Left-hand panels show responses for each neuron's preferred $(\mathrm{P}$, filled circles) and nonpreferred ( $\mathrm{N}$, unfilled circles) directions of motion; the panels on the right show the P-N difference (unfilled squares), indicating direction selectivity as a function of contrast. (A \& B) Response to varying carrier contrast, $C_{c}$ for two enveloperesponsive neurons. (C \& D) Response to varying envelope contrast, $C_{e}$, for the same neurons. average. However, there is clearly no systematic quantitative relationship between the two thresholds. In a similar manner, Fig. 6B plots the thresholds for envelope versus grating contrast in six cells for which both parameters could be reliably estimated. Once again the grating thresholds show considerable variability between neurons whilst those for the envelope are much more uniform. Although it was only possible to make comparisons between a very limited number of neurons, there does not appear to be any systematic relationship between the thresholds obtained for the two varieties of motion stimulus. Nonetheless, thresholds are again significantly lower for grating than for envelope contrast [Wilcoxon $z_{(6)}=-2.201 ; P<0.05$ ] by an average of about $1 \log$ unit.

Fig. 6C compares thresholds for second-order carrier and envelope contrast in the same neurons. All the data points are clustered in the upper right-hand corner, suggesting that the two types of second-order contrast threshold are closely coupled and similar in magnitude when measured in the same cells. The average ratio of carrier to envelope contrast thresholds is about 1.2, and the two kinds of threshold are not significantly different [Wilcoxon $z_{(7)}=-1.69 ; P>0.05$ ]. This result has important theoretical implications for current models of second-order motion processing and will be discussed below.

\section{Discussion}

Overview of results and relationship to previous studies

The direction selectivity of a cell is conventionally quantified by some metric comparing responses to opposing directions of motion at only one contrast level. Yet qualitative differences between the shapes of the CRFs measured for each direction could potentially manifest as a change in the cell's direction selectivity with con- 
(A)

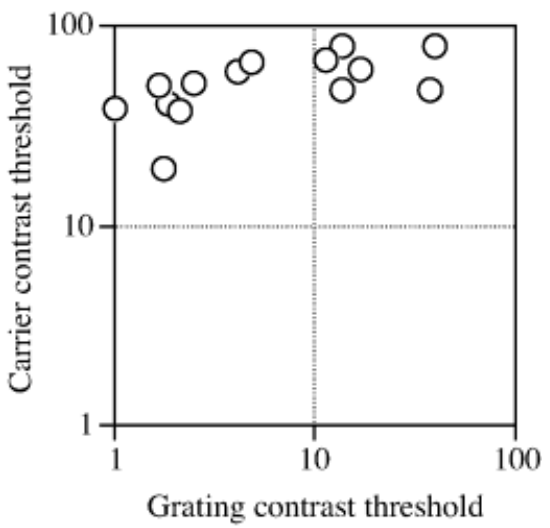

(B)

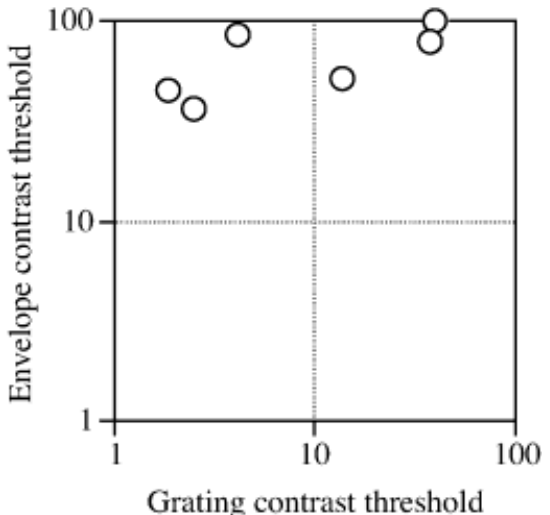

(C)

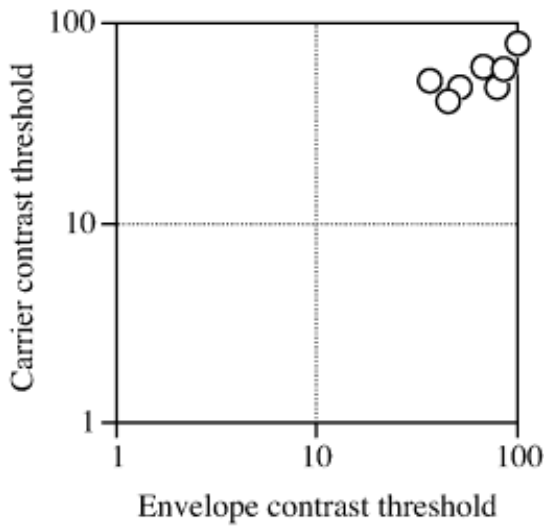

Fig. 6. Relationships between neurometric thresholds for three kinds of stimulus contrast, in neurons for which multiple kinds of measurement were available. In each scatterplot, each point represents neurometric thresholds ( $\alpha$ in the best-fitting Weibull function) for one neuron. (A \& B) Envelope-responsive cells exhibit relatively high thresholds for carrier (A) or envelope (B) contrast, across a much more broadly variable range of grating contrast thresholds. (C) Envelope-responsive neurons have similarly high thresholds for both carrier and envelope contrast.

trast. Regardless of the response metric adopted, the present study demonstrates that direction selectivity in cat area 18 often depends substantially on the contrast level tested.

Dean (1980) reported that for cat area 17 cells the CRF measured using sinusoidal gratings was approximately linear (when plotted on linear-linear axes) with contrast for both directions, with a shallower slope for the nonpreferred direction. At least for contrasts up to $25 \%$, there was no evidence of either response saturation or supersaturation. However, Li and Creutzfeldt (1984), testing over a wide range of contrasts, demonstrated contrastdependent directionality to drifting bar stimuli in cat area 17.

When tested with optimal gratings most neurons exhibited some degree of response compression and saturation at the highest contrasts tested, like those in previous studies of striate cortex (Albrecht \& Hamilton, 1982; Sclar et al., 1990; Albrecht \& Geisler, 1991; Geisler \& Albrecht, 1992, 1995; DeAngelis et al., 1993; Albrecht, 1995). However, some cells showed little response saturation whilst others exhibited supersaturation (the response peaked at a moderate contrast and fell at higher contrasts). Motion in the $\mathrm{N}$-direction often produced CRFs with different profiles to those in the P-direction, such that the magnitude of the $\mathrm{P}-\mathrm{N}$ difference response was contrast dependent. The P-N CRF was commonly a monotonic, sigmoidal function, but in $35 \%$ of cells it was nonmonotonic.

A few studies have noted supersaturation in the conventional CRF of some cat area 17 neurons (e.g., Albrecht \& Hamilton, 1982; Bonds, 1991, 1993; Peterson \& Freeman, 2003). Li and Creutzfeldt (1984) found that the CRF of cat area 17 neurons, measured using drifting bars, exhibited supersaturation in all 104 neurons investigated. Neurons' responses decreased by $5-40 \%$ at contrasts above that needed to produce response saturation (typically $>75 \%$ ). For the nine neurons in our study that showed P-direction supersaturation, the response decline occurred when the grating contrast was $80 \%$ for seven of the cells and $40 \%$ for the remaining two. Together these results suggest that supersaturation was not more prominent in most previous studies because it is most evident when the contrast range used to measure the CRF is very broad. For example, in the present study the maximum grating contrast tested was $80 \%$ and in $\mathrm{Li}$ and Creutzfeldt's study it was $\mathrm{ca} .90 \%$.

Quite unlike the heterogeneity in first-order responses, without exception the $\mathrm{P}-\mathrm{N}, \mathrm{P}$, and $\mathrm{N}$ second-order CRFs were always monotonic, regardless of whether carrier or envelope contrast was varied, even for those cells which showed a nonmonotonic P-N CRF to first-order motion. Thus, second-order direction selectivity did not generally exhibit the contrast-dependence seen for firstorder stimuli. This difference is unlikely to be due to our limited sample of second-order CRFs, since a sizeable proportion of cells exhibited some degree of nonmonotonic first-order response $(M I<$ 1.0 for $17 / 49 \mathrm{P}-\mathrm{N}$ CRFs). If this proportion (0.35) was a representative estimate of the general incidence of nonmonotonic response in the neuronal population as a whole, then not finding a single instance of nonmonotonic second-order response would be extremely unlikely $(P<0.001 ; n=16)$.

Weaker responses to second- than to first-order motion stimuli were also seen in previous studies of early cortical neurons (e.g. Zhou \& Baker, 1994), but to our knowledge the only other study comparing CRFs for first- and second-order motion was that of O'Keefe and Movshon (1998) examining neurons in area MT/V5. Comparing responses to sine-wave gratings and square-wave envelopes of dynamic versus static noise carriers, for preferreddirection motion, they found second-order responses only at higher carrier contrasts, and similar differences in CRF shapes for firstorder (luminance) versus second-order (carrier contrast) responses. While our second-order responses were generally also contingent upon high contrasts, we could rule out any explanation based on possible distortion artifacts of an early intensive nonlinearity (e.g., in the display screen, or the photoreceptors), due to the narrowband tuning to carrier spatial frequency $\left[f_{c}\right.$ in eqn. (2)] observed here and in previous studies (Zhou \& Baker, 1994; Mareschal \& Baker, 1999). 
Since the variance of a cortical neuron's response increases proportionately to its mean (Tolhurst et al., 1983), with a varying degree of dependence for different neurons and different time scales (Sachs \& Baker, 2003), the results of neurometric analysis might not be simply predictable from average differential responses. The resulting neurometric functions were usually (though not always) similar in shape to the P-N CRFs measured for the same cells, and demonstrate that the nonmonotonicity is significant in terms of information carried about direction of motion. Human psychophysical studies of motion perception have also demonstrated nonmonotonic dependence on contrast (Derrington \& Goddard, 1989; Cleary, 1990), though possibly for reasons not related directly to neuronal CRFs. Except for one cell, the second-order neurometric functions were also monotonic and neural contrast thresholds were, on average, $c a$. 10 times higher for second- than first-order motion.

\section{Different contrast response for first- and second-order motion: Neural mechanisms}

The heterogeneity of first-order CRFs suggests more than one underlying mechanism. While many neurons showed similar CRFs for P- and N-directions (Fig. 7D), over a third of the cells exhibited a nonmonotonic profile in the $\mathrm{P}-\mathrm{N}$ response to first-order motion.

About half of the cells that demonstrated a nonmonotonic $\mathrm{P}-\mathrm{N}$ CRF exhibited some degree of response supersaturation to motion in the P-direction (Fig. 4C), with negligible or monotonically rising $\mathrm{N}$-direction response (Figs. 7A-7B). If supersaturation ef-
(A)

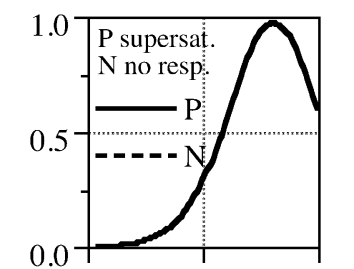

(B)

(C)

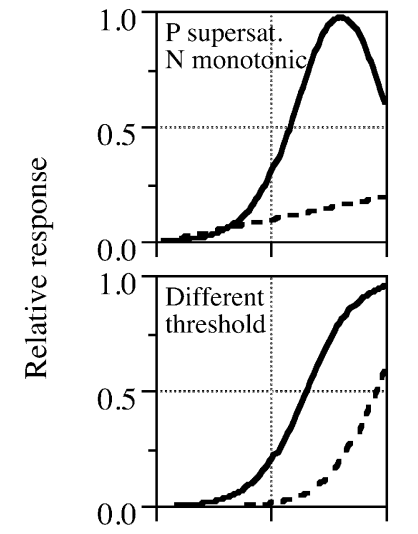

(D)

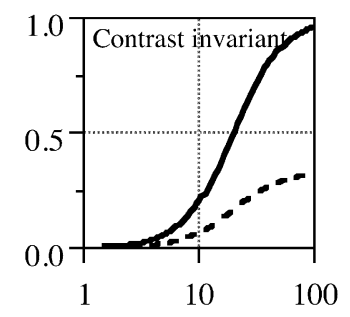

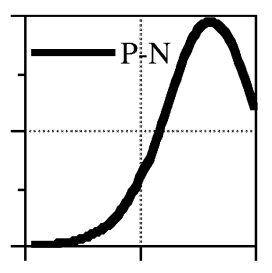
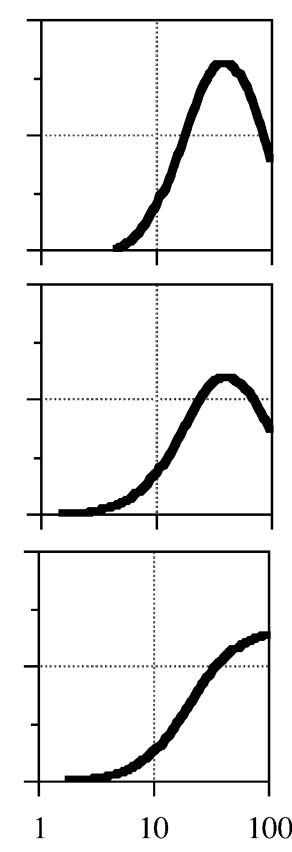

Grating contrast $(C)$
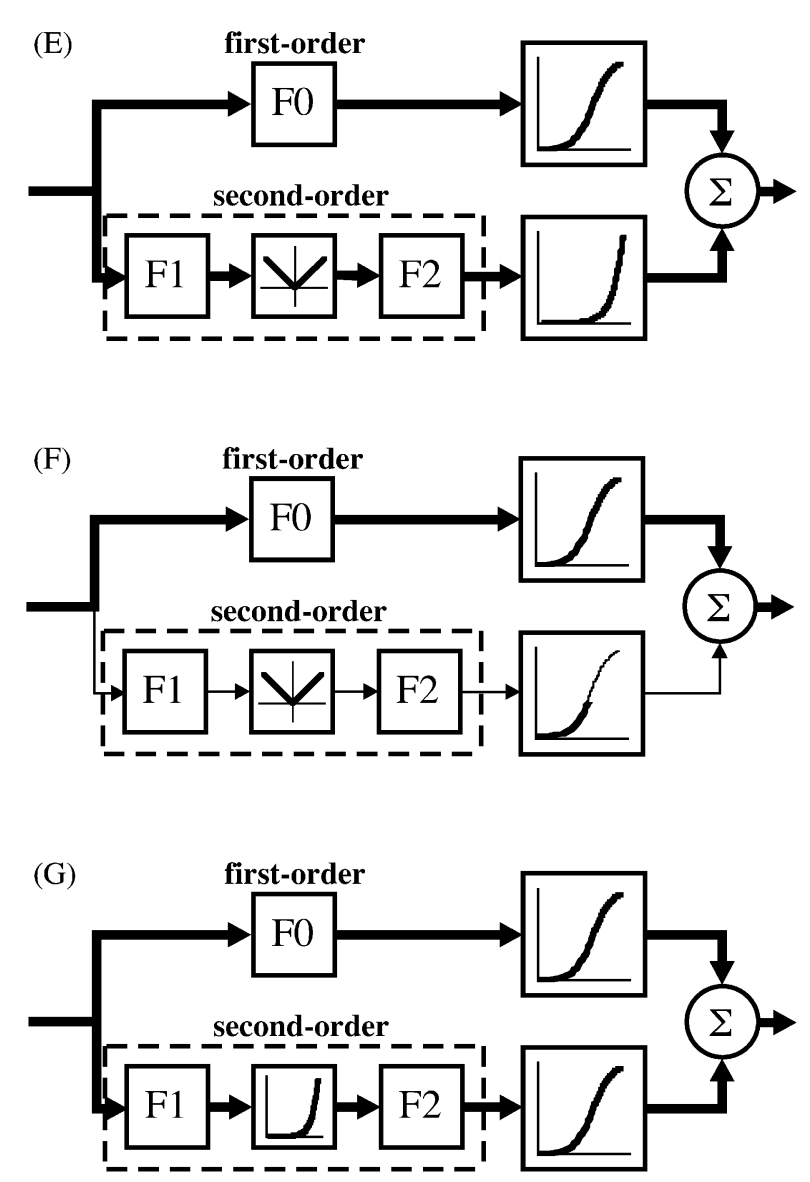

Fig. 7. (A-D) Schematic illustration of different ways in which responses in the $\mathrm{P}$ - and N-directions could combine to produce either a nonmonotonic or monotonic P-N contrast response function. (A) Supersaturation of the P-direction response (with no response in the N-direction) can give rise to a P-N CRF that is itself highly nonmonotonic. (B) Same as A, but the N-direction exhibits a monotonically increasing response as a function of contrast. (C) A nonmonotonic dependence of directionality on contrast can arise when there is a markedly different threshold for responding to opposing directions of motion. (D) Direction selectivity is monotonic when the change in firing rate with increasing contrast (i.e., upward slope of the CRF) in the N-direction does not exceed that of the P-direction, resulting in a P-N CRF that is monotonic. (E) Model depicting the cortical processing of first-order and second-order motion within two parallel processing streams, each specialized for encoding one type of motion (Zhou \& Baker, 1993). First-order motion is extracted by conventional motion sensors with receptive fields oriented in space-time (linear spatiotemporal filters [FO]). Second-order motion is extracted by a separate pathway in which the outputs of spatial frequency filters [F1] are subjected to a gross nonlinearity (e.g., rectification) prior to conventional motion analysis at a coarser spatial scale [F2]. In principle the processing streams could have qualitatively different contrast responses (E); or the same form of contrast response (F), but weaker signals from the second-order pathway only operate over the lower (convex) portion of the full response function (different signal strengths in the two pathways are represented by the thick $v s$. thin arrows). Another possibility is that the demodulation nonlinearity is not a simple rectification, but a steep power-law (G), as suggested by human psychophysics. 
fects are mediated by the same inhibitory mechanisms that govern response saturation (Bonds, 1993), a reduction in direction selectivity with increasing contrast could simply reflect a stronger inhibitory influence from cells with different tuning properties (lateral inhibition) or an "overshoot" of a divisive normalization that scales differently with contrast (Heeger, 1992b).

In most of the remaining cases of nonmonotonic contrastdependent directionality, a substantial $\mathrm{N}$-direction response was found only at the very highest contrasts (Fig. 7C). This pattern is not due to simple spike rate saturation, since the SI for the P-direction was not correlated with the maximum response (Fig. 4B). This decrease in direction selectivity with contrast might arise in conjunction with contrast-dependent changes in temporal properties, as documented in striate cortex (Holub \& Morton-Gibson, 1981; Dean \& Tolhurst, 1986; Carandini \& Heeger, 1994; Albrecht, 1995). Peterson and Freeman (2003) reported nonmonotonic contrast dependence of direction selectivity for many neurons in cat area 17, which they interpreted in terms of a disruption of temporal quadrature between lagged and nonlagged lateral geniculate nucleus (LGN) afferents (Humphrey \& Saul, 2002).

The qualitative difference in contrast response to the two varieties of motion is consistent with current models of cortical motion processing, suggesting that early cortical neurons receive distinct inputs from two independent processing streams, each encoding either first- or second-order motion (e.g., Zhou \& Baker, 1993). Within this scheme, second-order motion is detected by a more elaborate mechanism, for example an early linear filtering stage (F1), followed by a demodulating rectification ( $\mathrm{R})$ and then a second filtering at a lower spatial frequency (F2). The relative homogeneity of neurometric thresholds for second-order motion (Figs. 6B-6C), irrespective of whether the carrier or envelope contrast was varied, is also consistent with such a Filter-RectifyFilter (FRF) model of second-order motion processing (Fig. 7). As changing the contrast of either the carrier or the envelope of a second-order pattern will produce analogous changes in the magnitude of the (rectified) neural signal reaching F2, responses to the two types of second-order contrast will be closely coupled and neural thresholds similar.

The large difference in thresholds for first- versus second-order motion, and the much greater heterogeneity of the former, suggests that contrast is separately determined in the two streams. Perhaps the most straightforward interpretation is that the two processing streams have qualitatively different contrast response functions (Fig. 7E). On the other hand, the basic form of the contrast response to each variety of motion could be effectively the same, but the weaker responses in the second-order pathway might only modulate over the lower (convex) end of the full sigmoidal response function (differing signal strengths are indicated by the thick vs. thin lines in Fig. 7F). Another possibility is that the demodulation nonlinearity is not a simple rectification, but a steep power-law (Fig. 7G), as suggested in human psychophysics. This latter possibility has interesting functional implications that will be discussed below.

\section{Implications for sensory coding and perception}

The "efficient coding hypothesis", that sensory neurons respond to natural stimuli in ways that maximize the information conveyed (Barlow, 2001; Simoncelli, 2003), has been supported by demonstrations of neural CRFs whose shapes result in neural firing rates to natural stimuli which are optimally distributed (Laughlin, 1981; Baddeley et al., 1998). This idea suggests the possibility that the heterogeneity of first-order CRFs might reflect different categories of neurons specialized to efficiently represent functionally distinct kinds of natural image motion, which might have differing contrast distributions. A more specific implication of the large difference between first- and second-order CRFs is that the distribution of contrasts of first-order and second-order information might differ substantially in natural images. The high thresholds and steep slopes of second-order motion CRFs also suggest that they would provide a relatively more "binarized" or symbolic image representation; responses of these neurons to natural images would be highly selective and "sparse," a property which has important advantages for efficient sensory coding (Olshausen \& Field, 2004).

Our analysis has focused on spike rate responses of single neurons, with implications for underlying neural mechanisms. Neurometric analyses provide information about the potential informativeness of a neuron's firing about direction of motion, but motion perception is presumably mediated by population responses. While it is not clear how to properly pool individual neural responses into a behaviorally relevant population response, it is worth considering possible disparities between the two. For example, the data of $\mathrm{Li}$ and Creutzfeldt (1984) suggest that for the population of area 17 cells as a whole, direction selectivity is relatively invariant with changing contrast; however, for many of their individual neurons the CRF shapes for the $\mathrm{P}$ - and $\mathrm{N}$-directions were qualitatively different, and thus for individual neurons the degree of direction selectivity appeared to depend critically on the contrast level tested. Albrecht and Hamilton (1982) also noted that a population average CRF is not necessarily representative of individual neuron CRFs, at least in part because each cell has a limited dynamic range which differs substantially from one neuron to another. The varying degrees of saturation and supersaturation seen in our results suggest an increased variance of population response which might account for impaired psychophysical motion performance at high contrasts (Derrington \& Goddard, 1989; Cleary, 1990).

FRF models like those shown in Figs. 7E-7G have often been proposed not only for second-order motion, but also as models for human psychophysics of texture discrimination or segregation (e.g. Landy \& Graham, 2004). However, conventional forms of such models, employing a simple full-wave rectification or squarelaw as the demodulation nonlinearity, would fail to differentiate between certain textures having equal root-mean-square (RMS) contrast energy - for example, high versus low density of DCbalanced micropatterns (see Durgin \& Hammer, 2001). While such stimuli could be differentiated by yet another stage of rectification and filtering, a simpler modification to the FRF model would be a demodulation nonlinearity which is more nonlinear than full-wave rectification, for example a high threshold and/or a convex function (as in Fig. 7G). Other psychophysical studies of texture segregation (Graham \& Sutter, 1998) have supported a model with a more highly convex demodulation nonlinearity, such as a powerlaw device with exponent of $c a .3$ to 4 . Such a model (Fig. 7G) would naturally account for the highly convex second-order CRFs which we have described, as an alternative to the possibilities (Figs. 7E-7F) discussed earlier. If contrast envelope-responsive neurons were also responsive to boundaries between densitymodulated, RMS-balanced stimuli, it would favor a model like that of Fig. 7G, and would be consistent with such neurons providing a possible basis for perception of these stimuli.

\section{Acknowledgments}

We thank Linda Domazet for assistance with the experiments, and we are grateful to Rhone-Poulenc Rorer for donation of Gallamine triethiodide. 
We also thank Krystel Huxlin for helpful suggestions. Preliminary reports of some of these findings has been presented previously in abstract form (Ledgeway \& Baker, 2001). This research was supported by a Canadian Institutes of Health Research grant MA-9685 to C.L. Baker and in part by a grant from the University of Nottingham Research Committee to $\mathrm{T}$. Ledgeway.

\section{References}

Albrecht, D.H. (1995). Visual cortex neurons in monkey and cat: Effect of contrast on the spatial and temporal phase transfer functions. Visual Neuroscience 12, 1191-1210.

Albrecht, D.H. \& Geisler, W.S. (1991). Motion selectivity and the contrast response function of simple cells in the visual cortex. Visual Neuroscience 7, 531-546.

Albrecht, D.H. \& Hamilton, D.B. (1982). Striate cortex of monkey and cat: Contrast response function. Journal of Neurophysiology 48, 217-237.

Baddeley, R., Aвbot, L.F., Booth, M.C., Sengpiel, F., Freeman, T., WAKeman, E.A., \& Rolls, E.T. (1998). Responses of neurons in primary and inferior temporal visual cortices to natural scenes. Proceedings of the Royal Society B (London) 264, 1775-1783.

BAKER, C.L., JR. (1999). Central neural mechanisms for detecting secondorder motion. Current Opinion in Neurobiology 9, 461-466.

BARLOW, H.B., (2001). Redundancy reduction revisited. Network 12, 241-253.

Barlow, H.B. LeVICK, W.R. \& Yoon, M. (1971). Responses to single quanta of light in retinal ganglion cells of the cat. Vision Research 11, $87-101$.

Bonds, A.B. (1991). Temporal dynamics of contrast gain in single cells of the cat striate cortex. Visual Neuroscience 6, 239-255.

Bonds, A.B. (1993). The encoding of cortical contrast gain control. In Contrast Sensitivity, ed. Shapley, S. \& LAM, D.M.-K., pp. 215-230. Cambridge, Massachusetts: MIT Press.

Brainard, D.H. (1997). The psychophysics toolbox. Spatial Vision 10, 433-436.

Britten, K.H., Shadlen, M.N., Newsome, W.T. \& Movshon, J.A. (1992). The analysis of visual motion: A comparison of neuronal and psychophysical performance. Journal of Neuroscience 12, 4745-4865.

Brugge, J.F. \& Merzenich, M.M. (1973). Responses of neurons in auditory cortex of the macaque monkey to monaural and binaural stimulation. Journal of Neurophysiology 36, 1138-1158.

Carandini, M. \& HeEger, D. (1994). Summation and division by neurons in primate visual cortex. Science 264, 1333-1336.

Cavanagh, P. \& Mather, G. (1989). Motion: The long and short of it. Spatial Vision 4, 103-129.

Chaudhuri, A. \& Albright, T.D. (1997). A comparison of neuronal responses to edges defined by luminance or temporal texture in macaque area V1. Visual Neuroscience 14, 949-962.

Chubb, C. \& Sperling, G. (1988). Drift-balanced random stimuli: A general basis for studying non-Fourier motion perception. Journal of the Optical Society of America A 5, 1986-2007.

Cleary, R. (1990). Contrast dependence of short-range apparent motion. Vision Research 30, 463-478.

Cooper, G.F. \& Robson, J.G. (1968). Successive transformations of visual information in the visual system. In Pattern Recognition, IEEE N.P.L Conference publication, No. 42. London: IEEE.

DeAn, A.F. (1980). The contrast-dependence of direction selectivity. Journal of Physiology (London) 303, 38-39.

DeAN, A.F. (1981). The relationship between response amplitude and contrast for cat striate cortical neurons. Journal of Physiology 318, 413-427.

Dean, A.F. \& Tolhurst, D.J. (1986). Factors influencing the temporal phase of response to bar and grating stimuli for simple cells in the cat striate cortex. Experimental Brain Research 62, 143-151.

DeAngelis, G.C., Ohzawa, I. \& Freeman, R.D. (1993). Spatiotemporal organization of simple-cell receptive fields in the cat's striate cortex. II. Linearity of temporal and spatial summation. Journal of Neurophysiology 69, 1118-1135.

Derrington, A.M. \& Goddard, P.A. (1989). Failure of motion discrimination at high contrasts: Evidence for saturation. Vision Research 29, 1767-1776.

Durgin, F.H. \& Hammer, J.T. (2001). Visual aftereffects of sequential perception: Dynamic adaptation to changes in texture density and contrast. Vision Research 41, 2607-2617.
Foster, K.H., Gaska, J.P., NAgler, M. \& Pollen, D.A. (1985). Spatial and temporal frequency selectivity of neurones in visual cortical areas V1 and V2 of the macaque monkey. Journal of Physiology (London) 365, 331-363.

Geisler, W.S. \& Albrecht, D.G. (1992). Cortical neurons: Isolation of contrast gain control. Vision Research 32, 1409-1410.

Geisler, W.S. \& Albrecht, D.G. (1995). Bayesian analysis of identification performance in monkey visual cortex: Nonlinear mechanisms and stimulus certainty. Vision Research 35, 2723-2730.

Graham, N. \& SutTer, A. (1998). Spatial summation in simple (Fourier) and complex (non-Fourier) channels in texture segregation. Vision Research 38, 231-257.

HeEger, D.J. (1992a). Half-squaring in responses of cat striate cells. Visual Neuroscience 9, 427-443.

HeEgER, D.J. (1992 b). Normalization of cell responses in cat striate cortex. Visual Neuroscience 9, 181-197.

Holub, R.A. \& Morton-Gibson, M. (1981). Response of visual cortical neurons of the cat to moving sinusoidal gratings: Response contrast functions and spatiotemporal interactions. Journal of Neurophysiology 46, 1244-1259.

Hubel, D.H. \& Wiesel, T.N. (1968). Receptive fields and functional architecture of monkey striate cortex. Journal of Physiology (London) 195, 215-243.

Humphrey, A.L. \& Saul, A. B. (2002). The emergence of direction selectivity in cat primary visual cortex. In The Cat Primary Visual Cortex, ed. Payne, B.R. \& Peters, A., pp. 343-386. San Diego, California: Academic Press.

Landy, M.S. \& Graham, N. (2004). Visual perception of texture. In The Visual Neurosciences, ed. Chalupa, L.M. \& Werner, J.S., pp. 11061118. Cambridge, Massachusetts: MIT Press.

LAUGHLIN, S.B. (1981). A simple coding procedure enhances a neuron's information capacity. Zeitschrift fur Naturforschung C 36, 910-912.

Ledgeway, T. \& BAKer, C.L., JR. (2001). A neurometric function analysis of the direction selectivity of visual cortex neurons as a function of stimulus contrast. Society for Neuroscience Abstracts 27, 164.3.

Ledgeway, T., Zhan, C., Johnson, A., Song, Y. \& BaKer, C.L., Jr. (2004). Direction selectivity and the contrast response function of cortical neurones to first-order and second-order motion. Perception (Supplement) 33, 159-160.

Leventhal, A.G., Wang, Y., Schmolesky, M.T. \& Zhou, Y. (1998). Neural correlates of boundary perception. Visual Neuroscience 15, 1107-1118.

Li, C.-Y. \& Creutzfeldt, O. (1984). The representation of contrast and other stimulus parameters by single neurons in area 17 of the cat. Pflugers Archives 401, 304-314.

Mareschal, I. \& Baker, C.L., JR. (1998). Temporal and spatial response to second-order stimuli in cat area 18. Journal of Neurophysiology $\mathbf{8 0}$, 2811-2823.

Mareschal, I. \& BAKER, C.L. (1999). Cortical processing of second-order motion. Visual Neuroscience 16, 527-540.

Movshon, J.A. \& Tolhurst, D. (1975). On the response linearity of cells in the cat visual cortex. Journal of Physiology 249, 56-57.

O’ Keefe, L.P. \& Movshon, J.A. (1998). Processing of first- and secondorder motion signals by neurons in area MT of the macaque monkey. Visual Neuroscience 15, 305-317.

Olshausen, B.A. \& Field, D.J. (2004). Sparse coding of sensory inputs. Current Opinion in Neurobiology 14, 1-7.

Parker, A.J. \& Newsome, W.T. (1998). Sense and the single neuron: Probing the physiology of perception. Annual Review of Neuroscience 21, 227-277.

Pelli, D.G. (1997). The VideoToolbox software for visual psychophysics: Transforming numbers into movies. Spatial Vision 10, 437-442.

Peterson, M.R. \& Freeman, R.D. (2003). Direction selectivity of neurons in striate cortex changes with stimulus contrast. Abstract Viewer/ Itinerary Planner, Program No. 484.17. Washington, DC: Society for Neuroscience. Online.

Phillips, D.P., Semple, M.N., Calford, M.B., \& Kitzes, L.M. (1994) Level-dependent representation of stimulus frequency in cat primary auditory cortex. Experimental Brain Research 102, 210-226.

Robson, J.G. (1975). Receptive fields: Spatial and intensive representation of the visual image. In Handbook of Perception, Vol. 5: Vision, ed. Carterette, E.C. \& Friedman, M.P., pp. 81-112. New York: Springer-Verlag.

SACHS, A.J. \& BAKER, C.L., JR. (2003). Irregular firing patterns of visual 
cortex neurons behave like a Markov-modulated Poisson process. Journal of Computational Neuroscience (submitted).

Sclar, G., Maunsell, J.H.R. \& Lennie, P. (1990). Coding of image contrast in central visual pathways of macaque monkey. Vision Research 30, 1-10.

SimonCELLI, E.P. (2003). Vision and the statistics of the natural environment. Current Opinion in Neurobiology 13, 144-149.

Skottun, B.C., Bradley, A., Sclar, G., Ohzawa, I. \& Freeman, R.D. (1987). The effects of contrast on visual orientation and spatial frequency discrimination: A comparison of single cells and behaviour. Journal of Neurophysiology 57, 773-786.

Skottun, B.C., DeValois, R.L., Grosof, D.H., Movshon, J.A., AlBRECHT, D.G. \& Bonds, A.B. (1991). Classifying simple and complex cells on the basis of response modulation. Vision Research 31, 1079-1086.

Somers, D., DragoI, V. \& Sur, M. (2002). Orientation selectivity and its modulation by local and long-range connections in visual cortex. In The cat primary visual cortex, ed. PAyne, B.R. \& Peters, A., pp. 471520. San Diego, California: Academic Press.

Song, Y. \& BAKER, C.L., JR. (2003). Distinct linear and nonlinear mechanisms for illusory contour responses in visual cortex neurones. $A b$ - stract Viewer/Itinerary Planner, Program No. 910.10. Washington, DC: Society for Neuroscience. Online.

Tolmurst, D.J., Movshon, J.A. \& Dean, A.F. (1983). The statistical reliability of signals in single neurons in cat and monkey visual cortex. Vision Research 23, 775-785.

WeIbull, W.A. (1951). A statistical distribution function of wide applicability. Journal of Applied Mechanics, 21, 292-277.

Zhan, C., Ledgeway, T. \& BaKer, C.L., JR. (2002). Optical imaging of contrast-dependent direction selectivity in visual cortex. Abstract Viewer/ Itinerary Planner, Program No. 657.4. Washington, DC: Society for Neuroscience. Online.

ZHAN, C. \& BAKER, C.L., JR. (2003). Optical imaging of form-cue invariant orientation domains for illusory contours and contrast envelopes in visual cortex. Abstract Viewer/Itinerary Planner, program No. 910.8. Washington, DC: Society for Neuroscience. Online.

ZHou, Y.-X. \& BAKER, C.L., JR. (1993). A processing stream in mammalian visual cortex neurons for non-Fourier responses. Science $\mathbf{2 6 1}$, 98-101.

ZHOU, Y.-X. \& BAKER, C.L., JR. (1994). Envelope-responsive neurones in areas 17 and 18 of cat. Journal of Neurophysiology 72, 2134-2150. 